\title{
La curva de Phillips en México (1980-2019): ¿una muerte anunciada?
}

The Phillips Curve in Mexico (1980-2019), a death foretold?

A curva de Phillips no México (1980-2019): uma morte anunciada?

Ericka Judith Arias Guzmán

Universidad Nacional Autónoma de México, Facultad de Estudios Superiores Acatlán,

México

erickajudith@comunidad.unam.mx

https://orcid.org/0000-0002-1663-9509

Felipe Cruz Díaz

Universidad Nacional Autónoma de México, Facultad de Estudios Superiores Acatlán,

México

fel_economista@comunidad.unam.mx https://orcid.org/0000-0003-0600-6940

Karla Estefanía Barcellos Investigadora independiente karla.barcellos.eco@gmail.com

https://orcid.org/0000-0002-9861-7257 


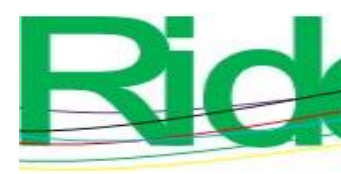

Revista Iberoamericana para la Investigación y el Desarrollo Educativo ISSN $2007-7467$

\section{Resumen}

La curva de Phillips ha sido utilizada para tomar decisiones importantes en la política monetaria. En el presente artículo, mediante un modelo estructural de vectores autorregresivos (SVAR por sus siglas en inglés), se estima la curva de Phillips para el caso de la economía mexicana durante el periodo 1980-2019. Pese a que algunos autores afirman que existe una relación inversa entre la tasa de desempleo y el nivel de los precios, dicha relación no es clara para la economía mexicana debido a que, desde finales de la década de los ochenta, la política monetaria ha estado dirigida a la estabilidad o control de precios sin que haya un resultado favorable en la generación de empleo.

Palabras clave: curva de Phillips, desempleo, inflación, modelo estructural de vectores autorregresivos, precios.

\section{Abstract}

The objective of this paper is to estimate the Phillips Curve for the case of the Mexican economy to the period 1980-2019 using a structural model of autoregressive vectors (SVAR). This Curve has been the subject of great debate as to the importance it has for monetary policy decision-making in the world economies. Some authors agree with the statement that there is an inverse relationship between the unemployment rate and the Price level. However, this relationship is not clear for the Mexican economy due to the fact that since the end of the eighties, monetary policy has been directed at controlling prices without a favorable result in the generation of employment.

Keywords: Phillips Curve, unemployment, inflation, SVAR model, prices.

\section{Resumo}

A curva de Phillips tem sido usada para tomar decisões importantes na política monetária. Neste artigo, usando um modelo de vetor estrutural autorregressivo (SVAR), a curva de Phillips é estimada para o caso da economia mexicana durante o período 1980-2019. Embora alguns autores afirmem que existe uma relação inversa entre a taxa de desemprego e o nível de preços, essa relação não é clara para a economia mexicana porque, desde o final dos anos 1980, a política monetária tem sido voltada para a estabilidade ou controle de preços sem um resultado favorável em. criação de emprego. 


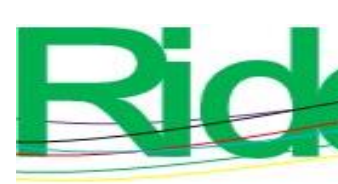

Revista Iberoamericana para la Investigación y el Desarrollo Educativo ISSN 2007-7467

Palavras-chave: curva de Phillips, desemprego, inflação, modelo estrutural vetorial autorregressivo, preços.

Fecha Recepción: Noviembre 2020

Fecha Aceptación: Julio 2021

\section{Introducción}

W. Phillips (1958) demostró que existía una relación inversa entre el crecimiento de los salarios monetarios (y los precios) y la tasa de desempleo para el caso del Reino Unido. Por eso, para el análisis de la relación de estas variables se utilizó el inverso de la tasa de desempleo como indicador del exceso de demanda laboral con el fin de exponer la posibilidad de que la inflación sea considerada como una variable en la estimación del nivel de desempleo y, por lo tanto, del producto (Mántey, 1997).

El éxito de la curva de Phillips se debe a la capacidad analítica que presenta para ajustarse a diferentes teorías que se han desarrollado sobre la inflación. En algunas investigaciones se estimó para otros países y se obtuvieron resultados similares a los del Reino Unido, y fueron interpretados como indicadores de la potencialidad de las políticas monetaria y fiscal para acelerar el crecimiento (Rodríguez, Ludlow y Peredo, 2004).

Las estimaciones en esos países fueron utilizadas para la toma de decisiones respecto a la política económica con el propósito de establecer objetivos de inflación y desempleo estables que permitieran impulsar el nivel de producto.

En este sentido, suponían que el comportamiento de dicha curva sería vertical en el largo plazo, lo que significaba que a una tasa natural de desempleo (NAIRU por sus siglas en inglés) la inflación permanecería constante debido a que coincidiría con el pleno empleo de los factores productivos (Dorta, Álvarez y Zavarce, 2001).

Con el propósito de realizar estimaciones en las diferentes economías y para facilitar su planteamiento, en la curva de Phillips se sustituyó la ecuación de salarios por una de precios. De esta forma, a partir de la pendiente de dicha curva se podrían derivar los niveles de desempleo compatibles con una determinada meta de inflación. Actualmente, el debate se centra en la afirmación de que, para cada economía, existe un nivel de empleo o de desempleo que no necesariamente acelera la inflación.

El objetivo de este trabajo, por ende, es estimar la curva de Phillips para la economía mexicana en el periodo de 1980-2019 mediante un modelo SVAR. Para ello, el presente trabajo se divide en tres apartados. 

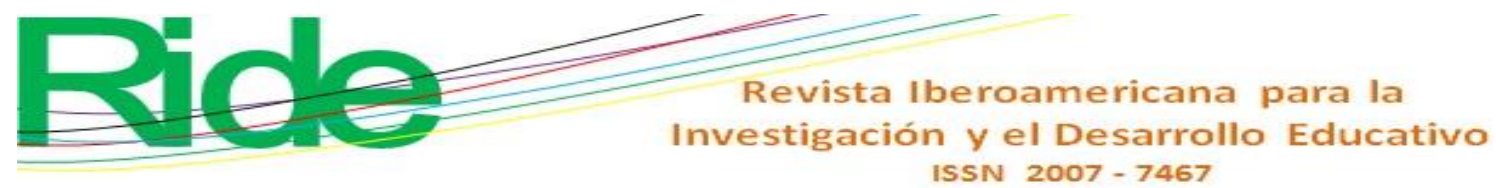

En el primero se hace una breve presentación del desarrollo teórico-histórico de la discusión sobre la curva de Phillips, así como de los resultados empíricos para algunos países; en el segundo apartado, dado que una de las variables de la curva de Phillips es la inflación, se analiza la relación entre la tasa de interés como objetivo intermedio de política monetaria y el tipo de cambio. Esto para alcanzar la estabilidad de precios en México que se ha aplicado desde la década de los ochenta. Por último, en el tercer apartado se presenta la metodología y el modelo econométrico SVAR que se utilizó para estimar la curva de Phillips en el caso de México durante el periodo 1980-2019.

\section{Desarrollo teórico-histórico de la discusión y evidencia empírica sobre la curva de Phillips}

La publicación del trabajo de Phillips en 1958, titulado The relation between unemployment and the rate of change of money wages in the United Kingdom, originó un gran debate que sigue vigente. El análisis de Phillips parte del supuesto de que en el mercado de trabajo existe escasez de mano de obra, lo que implica que para aumentar el nivel de producto se deberá aumentar el nivel de salarios nominales, lo que a su vez incrementará la tasa de salarios; por el contrario, cuando el desempleo aumenta, la tasa de salarios disminuye lentamente, lo que determinaría una relación inversa entre desempleo y la tasa de cambio de los salarios (Phillips, 1958).

\section{Desarrollo teórico-histórico de la curva de Phillips}

Bajo el marco analítico presentado en la sección anterior, Phillips realizó un estudio empírico para Inglaterra en el que consideró la relación entre las variables salario monetario y desempleo para un periodo de 96 años. Esto lo dividió en diferentes etapas delimitadas por los diversos procesos histórico-económicos que se vivieron durante ese tiempo. El modelo que estimó fue una regresión por el método de mínimos cuadrados ordinarios de la siguiente forma:

$$
Y+a=b X^{c}
$$

que al aplicarle una transformación logarítmica quedó de la siguiente forma:

$$
\log (Y+a)=\log b+\operatorname{cog} X
$$

donde $Y$ es la tasa de cambio de los salarios; $X$ es el porcentaje del desempleo, y $a, b$ y $c$ son constantes. 


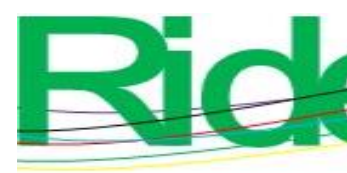

Revista Iberoamericana para la Investigación y el Desarrollo Educativo ISSN 2007 - 7467

En este estudio se llegó a la conclusión de que la tasa de cambio del desempleo determina la tasa de los cambios monetarios, pero se señala que esta conclusión es relativa, ya que debería profundizarse en el análisis de la relación entre el desempleo, la tasa de salarios, los precios y la productividad (Phillips, 1958).

Por otro lado, como se menciona en Rodríguez et al. (2004), Lipsey (1960) argumentó que los cambios en los precios correspondían al comportamiento de los salarios, de tal forma que la curva se reformuló en términos de la relación inversa entre la tasa de cambio de los precios y la tasa de desempleo. Por su parte, Samuelson y Solow (1960) realizaron un estudio similar para la economía de Estados Unidos considerando el periodo 1900-1960, donde parten de las aportaciones de Lipsey (1960) y llegan nuevamente a la relación inversa entre la inflación y el desempleo detectada previamente en las pruebas empíricas de Phillips en 1958. Fue a partir de esta confirmación que a la relación entre las tasas de inflación y desempleo se le denominó curva de Phillips.

Por su parte, Friedman (1967) y Phelps (1968) contribuyeron a la discusión de la curva de Phillips al retomar el argumento clásico donde el equilibrio en el mercado de trabajo depende del salario real, y no del salario nominal. De esta forma, un exceso de demanda en el mercado de trabajo se resuelve mediante el incremento del salario real, y no del nominal. Partiendo del concepto de tasa natural aplicado por Wicksell al tipo de interés, estos economistas postularon la presencia de una tasa natural de desempleo para afirmar que una reducción del desempleo mediante políticas expansivas provocaría mayores tasas de inflación.

Esta nueva concepción de la curva tradicional de Phillips prevaleció hasta la década de los años setenta cuando se incorporó en el debate el análisis de las expectativas como otro factor determinante de los precios. A esta curva se le denominó curva de Phillips aumentada con expectativas.

Posteriormente, el desarrollo de las expectativas racionales en los trabajos de Muth (1961), Lucas (1973), Sargent (1973) y Sargent y Wallace (1975) contribuyeron a reforzar el enfoque de Friedman-Phelps al afirmar que el comportamiento racional de los agentes económicos les permitía anticipar el efecto inflacionista de la política expansiva, de tal forma que la incorporación de estas expectativas a sus negociaciones salariales aseguraba que el salario real se mantuviera en equilibrio. Esta afirmación implicaba que la curva de Phillips fuera vertical, con lo que la discusión minimizó el impacto de la política fiscal para 


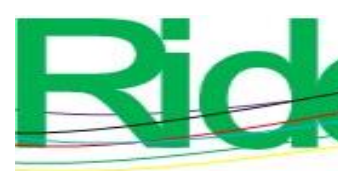

Revista Iberoamericana para la Investigación y el Desarrollo Educativo ISSN $2007-7467$

estimular el crecimiento del producto y el empleo (esto argumentando que cualquier incremento en el gasto de gobierno generaría un efecto inflacionario sin ningún efecto en el nivel del producto a largo plazo).

Por otro lado, con la aportación de James Tobin (1972) se elaboró una ecuación de precios y otra de salarios, y se centró en el análisis de los efectos de la relación salariosprecios sobre la dinámica de la oferta agregada. Al respecto, concluye que un cambio en la productividad del trabajo como consecuencia de movimientos en el desempleo es un componente que apoya la idea de un markup constante útil para explicar los movimientos acíclicos en el salario.

En la actualidad la discusión en torno a la curva de Phillips ha retomado importancia debido a que algunos economistas consideran que es un instrumento importante en el diseño y la toma decisiones de política económica.

En el caso de la escuela neokeynesiana, su análisis se centra en el carácter de la no linealidad de dicha curva. Al respecto, en los trabajos de Calvo (1983), Schaling (1999), Mankiw (2001) y Colombo y Weinrich (2003) se sostiene que la rigidez de precios impide que se cumpla el equilibrio clásico del mercado de trabajo.

En la investigación realizada por Okun (1962) se introduce un elemento analítico importante en la curva de Phillips, ya que analiza la relación entre el desempleo y el producto a partir del concepto de brecha del producto (output gap) en lugar de brecha en el desempleo (ley de Okun). La primera representa la diferencia entre los niveles de producto observado y el potencial. El producto potencial se mide mediante el nivel de producto consistente a una tasa de inflación determinada que está en función del capital productivo en existencia; la segunda representa las desviaciones observadas de la tasa de desempleo respecto a la tasa natural. Esta tasa es la que se mantiene cuando se incorporan los precios y salarios, y también se le conoce como NAIRU.

Desde hace varias décadas, la curva de Phillips ha sido utilizada por los tomadores de decisiones de política económica y particularmente de política monetaria, ya que mediante su estimación se fija la meta inflacionaria. En este sentido, se afirma que la tasa de inflación será estable siempre y cuando el producto observado sea igual al producto; de lo contrario, si el nivel de producto observado está por encima del producto potencial, la autoridad monetaria intervendrá para disminuir las presiones inflacionarias. Por otra parte, 


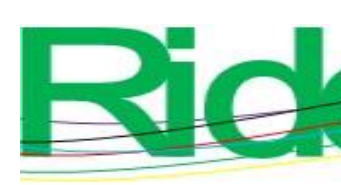

Revista Iberoamericana para la Investigación y el Desarrollo Educativo ISSN 2007-7467

si el nivel de producto observado se encuentra por debajo del producto potencial, se podrá estimular el crecimiento del producto sin comprometer en nivel de precios.

Desde una perspectiva keynesiana, el nivel de inflación es determinado por el nivel de output gap, ya que se supone que un aumento de la inflación es resultado de una reducción de este, a pesar de que el nivel de producto no crezca por encima del producto potencial. En efecto, se estima que el incremento en los precios puede ser más pronunciado en la fase creciente del ciclo económico que en la desaceleración observada cuando el output gap es negativo. Esta idea se fundamenta en la posición keynesiana de que la curva es casi vertical cuando está cerca del nivel del producto potencial (Laguna, 2007).

Por otro lado, Fisher argumenta que los tipos de interés nominales son indicadores de las expectativas de inflación y, por lo tanto, una herramienta útil para lo que denomina como la correcta aplicación de la política monetaria. En este sentido, si se considera que el Banco Central tiene como objetivo estabilizar la tasa de inflación en el mediano y largo plazos, es fundamental que la autoridad monetaria conozca la expectativa de inflación de los agentes, y si el efecto Fisher $^{l}$ se cumple, los tipos de interés nominales se convertirán en el instrumento ideal para esa correcta aplicación de la política monetaria.

\section{Evidencia empírica sobre la existencia de la curva de Phillips}

López y Misas (1999) estimaron la curva de Phillips lineal en Colombia, donde presentaron evidencia empírica sobre la naturaleza del trade-off entre el producto e inflación en dicho país, y mostraron las posibilidades que existen para mejorar la estimación de una curva de Phillips lineal.

Otros trabajos como el de Muñoz, Rojas, Sáenz y Tenorio (2003) estimaron la curva de Phillips aumentada con expectativas para Costa Rica mediante el método de corrección de errores, aplicando una técnica sugerida por Engle y Granger (1987). El estudio concluye que el $90 \%$ del comportamiento de la inflación fue resultado del comportamiento de la tasa de devaluación nominal, la tasa de inflación externa, la brecha en la producción y la inflación rezagada.

Por su parte, Morales (2004) estimó un modelo VAR para investigar por el lado de la oferta el efecto de los factores monetarios sobre la inflación en la zona del euro. A partir de los resultados obtuvieron tres conclusiones: 1) una importante persistencia de la

\footnotetext{
${ }^{1}$ Un aumento en el tipo de interés nominal provocará un incremento en la tasa de inflación futura.
} 


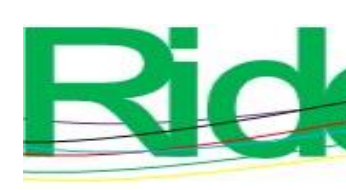

Revista Iberoamericana para la Investigación y el Desarrollo Educativo ISSN 2007 - 7467

inflación en la zona del euro; 2) los costos internos son una variable fundamental determinante de la inflación, por el lado de la oferta de las economías de la zona del euro, y 3) la inflación en el corto plazo tuvo un comportamiento cíclico. El coeficiente estimado para el mecanismo de corrección de errores es muy pequeño. Esto implica que los precios se ajustan muy lentamente ante variaciones, por lo que el equilibrio se reestablece de forma paulatina (Laguna, 2007).

Rico (1999) analizó el efecto Fisher y la paridad del interés real para la economía española de 1982 a 1998 utilizando la metodología de series de tiempo, particularmente el procedimiento de Johansen para diferenciar los efectos de corto y largo plazos. El trabajo concluye que el efecto Fisher se cumple en el largo plazo ajustado por los impuestos, y no por la paridad del interés real. Durante el periodo considerado, los tipos de interés anticiparon el comportamiento futuro de la tasa de inflación y fueron utilizados para fijar los objetivos de inflación de largo plazo. A pesar de dicho efecto, no se cumplió para el corto y mediano plazo, y el tipo de interés influyó en las decisiones de consumo e inversión.

En el trabajo de Rodríguez et al. (2004) se realizaron estimaciones para México con datos mensuales de 1987 a 2003 y la curva de Phillips ampliada con expectativas adaptativas de la siguiente forma:

$$
\pi_{t}=\beta_{1} \pi_{t-1}-\beta_{2}\left(u_{t}-u^{*}\right)+v
$$

donde, $\pi \mathrm{t}$ es la inflación actual, $\pi \mathrm{t}-1$ la inflación pasada, $v$ el término de error y $\mathrm{u}^{*}$ como una constante.

Los resultados obtenidos en este estudio presentaron dificultades de tipo estadístico debido a que la inflación se comportó de forma estacionaria, y el desempleo fue no estacionario; se consideró una función de tipo escalonada para obtener una estimación correcta y estadísticamente robusta.

Por su parte, las estimaciones numéricas de la tasa de desempleo muestran que es más alta en periodos donde la inflación es elevada; no obstante, el desempleo observado resultó menor a las tasas NAIRU. Esto es consecuencia de la contención de los salarios que ha impulsado la autoridad mexicana desde la década de los ochenta por el objetivo de metas inflacionarias que ha deprimido a la demanda agregada. 


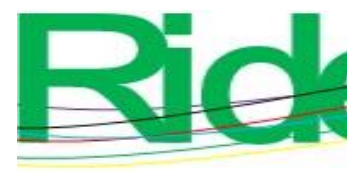

Revista Iberoamericana para la Investigación y el Desarrollo Educativo ISSN $2007-7467$

Posteriormente, estos mismos autores, en 2006, propusieron una ecuación y medición del nivel de desempleo utilizando a la curva de Phillips como marco conceptual para países latinoamericanos (Argentina, Brasil, Colombia, Chile y México) partiendo del supuesto de estabilidad de precios en el marco de la liberalización económica y financiera.

Estimaron un modelo de datos de panel para estos países y concluyeron que sí hay evidencia para configurar la curva de Phillips; sin embargo, reconocieron que el modelo es robusto debido a que con otras especificaciones no se obtiene la relación inversa entre inflación y desempleo.

Por el contrario, en el trabajo de Loría, Valdez y Tirado (2019) se estima la NAIRU mediante el modelo generalizado de momentos con la metodología de Ball y Mankinw (2002). Con esto demostraron que la relación entre desempleo e inflación se ha fortalecido en los últimos años.

\section{La política monetaria en México. La tasa de interés como objetivo intermedio de la política monetaria y el tipo de cambio como mecanismo de transmisión de la política monetaria}

A partir de 1982, se instrumentó en México la estrategia de banda con el propósito de mantener un tipo de cambio nominal subvaluado para impulsar las exportaciones y promover la sustitución de importaciones que provocaría que el déficit de la cuenta corriente de la balanza de pagos se mantuviera estable con el aumento en el nivel de exportaciones. Sin embargo, el diseño de la política monetaria estaba subordinado al efecto inflacionario de la devaluación debido a que mientras más elevado fuera el margen de subvaluación, mayor sería la presión sobre los precios. En efecto, se esperaba que, con un superávit en la balanza comercial y altas tasas de interés reales, los principales componentes de la demanda agregada (consumo e inversión) disminuirían, reduciendo con esto las presiones en los precios. En este sentido, mientras el tipo de cambio subvaluado permitía enfrentar las presiones externas, la política de altas tasas de interés alimentaba las presiones sobre los precios. Ante esta situación, la devaluación del peso fue inminente a finales de 1987 con el consecuente incremento en el nivel de los precios.

A partir de 1988, el tipo de cambio se adoptó como ancla nominal que buscaba influir en las expectativas de inflación, para lo cual se implementó el mecanismo de banda cambiaria que permitiera mantener el tipo de cambio nominal estable, como se observa en 

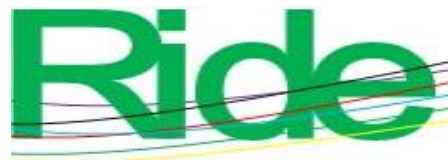

la figura 1. La estabilidad del tipo de cambio se logró mediante las operaciones de esterilización, mientras que las variaciones en la liquidez se compensaban a través del crédito interno neto (Cruz, 2006).

Figura 1. Tipo de cambio nominal e inflación, 1980-2019 (promedios mensuales)

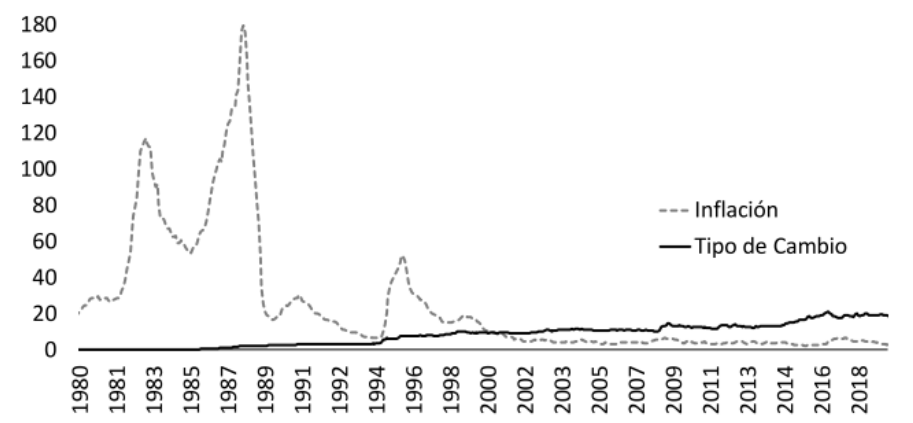

Fuente: Elaboración propia con datos del Inegi y del Banco de México

La figura 2 muestra como a partir de la década de los años noventa la deuda interna es significativa. Particularmente en el 2001 inicia un periodo de crecimiento como una medida de política monetaria para contener la inflación. En efecto, la deuda se utiliza como mecanismo compensatorio para mantener con tendencia creciente el nivel de las reservas internacionales con el propósito de estabilizar la inflación y lograr el objetivo de política monetaria. 


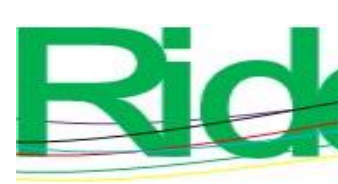

Revista Iberoamericana para la
Investigación y el Desarrollo Educativo
ISSN $2007-7467$

Figura 2. Deuda interna y cetes, 1990-2019 (cierres anuales y variaciones)

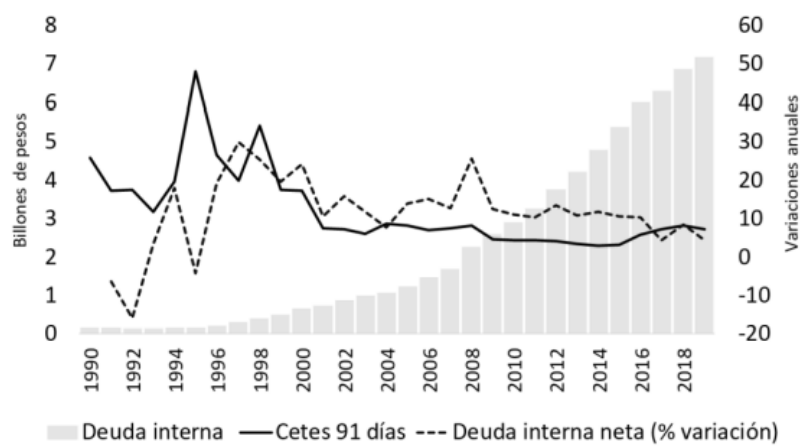

Fuente: Elaboración propia con datos del INEGI y de la Secretaría de Hacienda y Crédito Público

En los últimos años, debido al comportamiento de la inflación y la necesidad de equiparar los tipos de interés internos con los internacionales, el Banco de México se ha visto obligado a utilizar medidas monetarias restrictivas para lograr el objetivo de estabilidad de precios. La tendencia que muestran el tipo de cambio y la tasa de inflación, así como el comportamiento de las tasas de interés internacionales han influido en el manejo y diseño de la política monetaria. Si bien la tasa de interés de corto plazo influye en las primeras decisiones de ahorro e inversión, es cierto que en el caso de México el tipo de cambio es el principal mecanismo de transmisión de la política monetaria (Levy, 2001).

Lo anterior explica la razón por la que, desde finales de la década de los ochenta, el Banco de México ha utilizado las tasas de interés como instrumento intermedio de política monetaria y al tipo de cambio como mecanismo estabilizador de los precios.

Al respecto, varios estudios realizados para el caso de México han demostrado la estrecha relación que existe entre la tasa de devaluación y la tasa de inflación (Mántey, 2005; Ros, 1979), es decir, el mecanismo de transmisión de la política monetaria se sustenta en el tipo de cambio.

En la figura 3 se muestra la estrecha relación que existe entre la tasa de subvaluación o sobrevaluación y la tasa de inflación, y cómo ambas muestran la misma tendencia, lo que sugiere una cointegración en el largo plazo. 


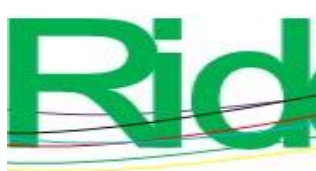

Revista Iberoamericana para la Investigación y el Desarrollo Educativo ISSN 2007 - 7467

Figura 3. Índice de sub/sobrevaluación del tipo de cambio e inflación anual, 1980-2019

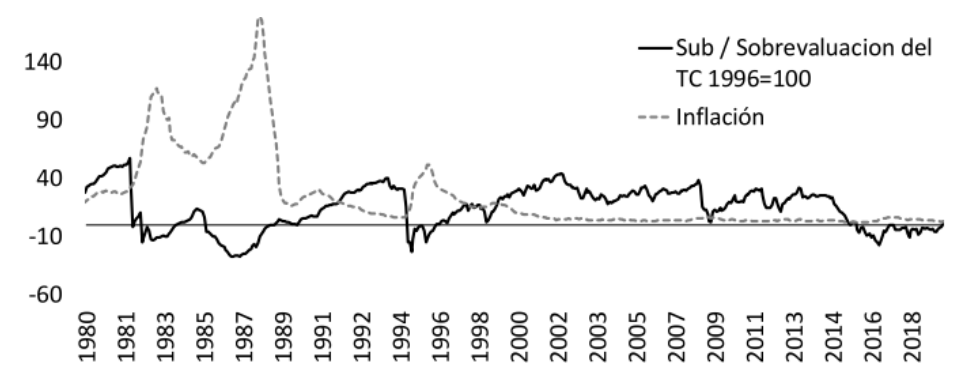

Fuente: Elaboración propia con datos del INEGI, del Banco de México y del Federal Reserve Bank of St. Louis, EE. UU.

Sobrevaluación o subvaluación se trata de la diferencia en puntos porcentuales que hay entre el tipo de cambio nominal y el tipo de cambio teórico. En el presente ejercicio el año base es 1996. El signo negativo muestra subvaluación del peso respecto al dólar y, por el contrario, el signo positivo revela sobrevaluación.

Por su parte, durante el periodo de 1980 a 2019, la curva de Phillips en México ha mostrado una pendiente positiva (figura 4). Esto significa que a medida que aumenta la inflación, el desempleo es mayor. Las tasas de inflación más elevadas y de mayor duración en México ocurrieron en la década de los años ochenta: en 1982 por la crisis de deuda; en 1987 debido los problemas originados por la caída en la Bolsa de Valores, así como las presiones a nivel internacional; en 1995 por la crisis financiera; y en 2009 por la crisis inmobiliaria originada en Estados Unidos. En la figura 4 se puede observar el comportamiento de estas variables, resaltando que presentan la misma tendencia, es decir, que existe una relación positiva entre la tasa de desempleo y la inflación.

Figura 4. Inflación y desempleo en México 1987-2019

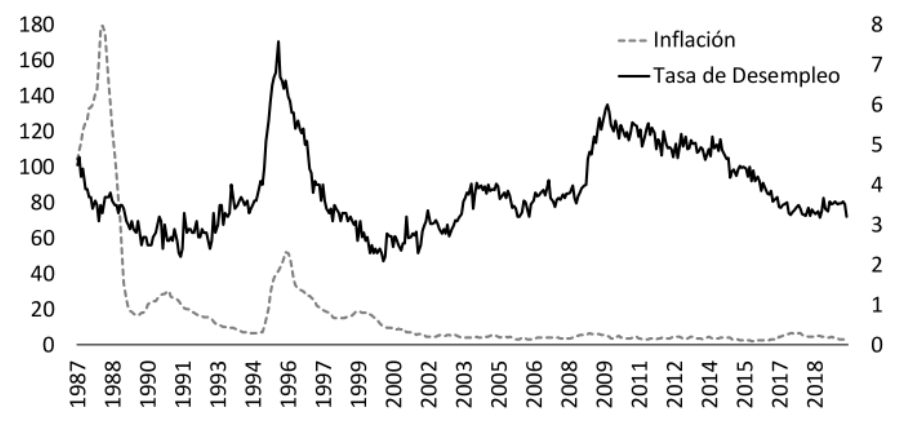

Fuente: Elaboración propia con datos del INEGI y la OCDE

Durante los últimos veinte años, aunque no es claro, también se puede observar una relación entre las variables, por lo que se podría deducir que la relación se da en periodos 


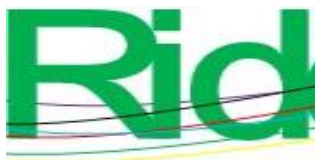

Revista Iberoamericana para la Investigación y el Desarrollo Educativo ISSN 2007 - 7467

cortos, pero no en el largo plazo ni de manera sostenida (ver las gráficas de correlación, figura 5).

Figura 5. Curva de Phillips en México, periodos 1980-2019 y 2008-2019
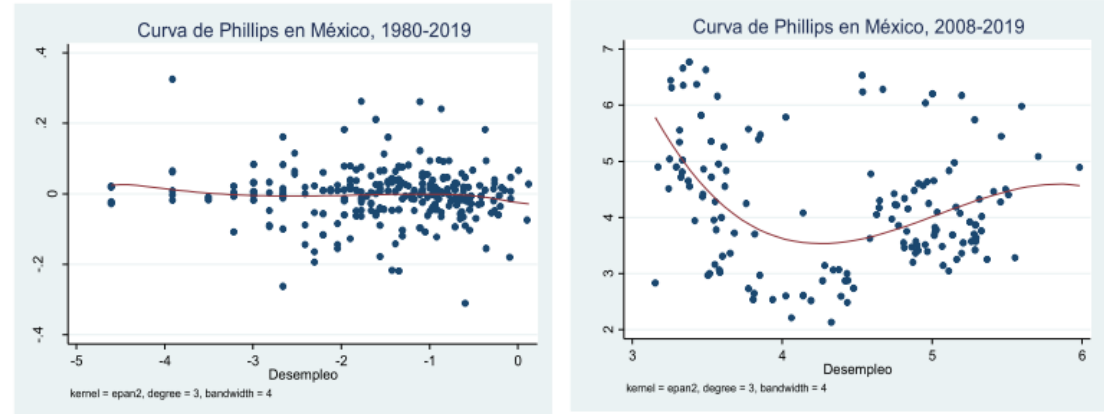

Fuente: Elaboración propia con información del modelo

En la figura 6 se muestra la relación que existe entre el salario mínimo real, es decir, el salario mínimo deflactado y el desempleo. Cabe señalar que la relación inversa entre salarios y desempleo que originalmente argumentó Phillips para el caso de México se observa en el periodo de 1980 a 1995; sin embargo, en los siguientes años existe un claro desencadenamiento entre estas variables.

Figura 6. Desempleo y salario real, 1980-2019

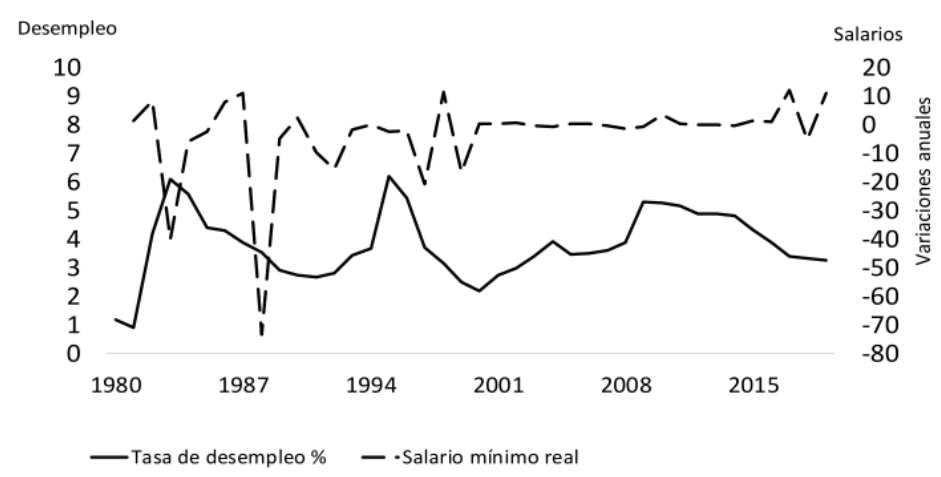

Fuente: Elaboración propia con datos del INEGI y la Secretaría de Hacienda y Crédito Público 


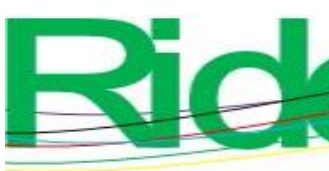

Revista Iberoamericana para la Investigación y el Desarrollo Educativo ISSN 2007-7467

\section{La vigencia de la curva de Phillips en México: estimación mediante un modelo SVAR}

La metodología de SVAR se estima cuando las variables analizadas no se encuentran integradas entre sí y las series de datos son estacionarias (Schenck, 2016). Estos tienen como objetivo definir respuestas dinámicas en las variables económicas que se analicen, haciendo uso de la información contenida en las series de tiempo y la teoría económica (Ramos, 2000).

Los modelos VAR tradicionales se utilizan en variables que se explican a sí mismas, es decir, en variables endógenas. En algunos casos con regresores deterministas, pero no integran dentro del modelado a la teoría económica (Amisano y Giannini, 1997). Las ventajas de los modelos SVAR son que, además de establecer las respuestas dinámicas, permiten el modelado explícito que identifica la interdependencia entre las variables (Forni y Gambetti, 2014), resaltando el análisis de la descomposición de la varianza y las funciones de impulso respuesta.

Para realizar este tipo de ejercicios, se utiliza la metodología SVAR, con la que se recuperan los parámetros estructurales originales a través de combinaciones de

perturbaciones estructurales que, en nuestro caso, se presentaron mediante choques de política monetaria y cambiaria que permiten definir las restricciones de corto y largo plazo en nuestros modelos.

\section{Base de datos}

En una primera aproximación, en este estudio se analizan las series mensuales de las tasas de cambio de la inflación y el desempleo para México con el fin de observar la relación existente entre ellas en el largo plazo para el periodo de 1980 a 2019.

En un segundo modelo se agregan las variables tipo de cambio y tasa de interés con el fin de identificar las diferencias en la dinámica establecida en el primer modelo. Para el segundo modelo, el periodo se acota a los años de 2008 a 2019 para contar con datos homogéneos publicados por el Instituto Nacional de Geografía y Estadística en la Encuesta Nacional de Ocupación y Empleo (ENOE), así como para tomar en cuenta el lapso en el que el Banco de México ha implementado la tasa de interés objetivo como parte de la política monetaria en el país. 

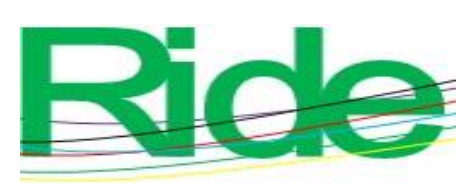

Revista Iberoamericana para la Investigación y el Desarrollo Educativo ISSN 2007 - 7467

Para las estimaciones econométricas se empleó información de variables macroeconómicas publicadas por el Instituto Nacional de Geografía y Estadística (INEGI), el Banco de México y la Organización para la Cooperación y el Desarrollo Económicos. Las variables fueron obtenidas en tasas y fueron transformadas a logaritmo natural y, en algunos casos, a primeras diferencias. Los datos de las series son mensuales y cumplen con el principio de estacionariedad básico y fundamental para su modelado econométrico (ver figura 7).

Figura 7. Análisis de las series en tasas y pesos. En logaritmos naturales y primeras diferencias
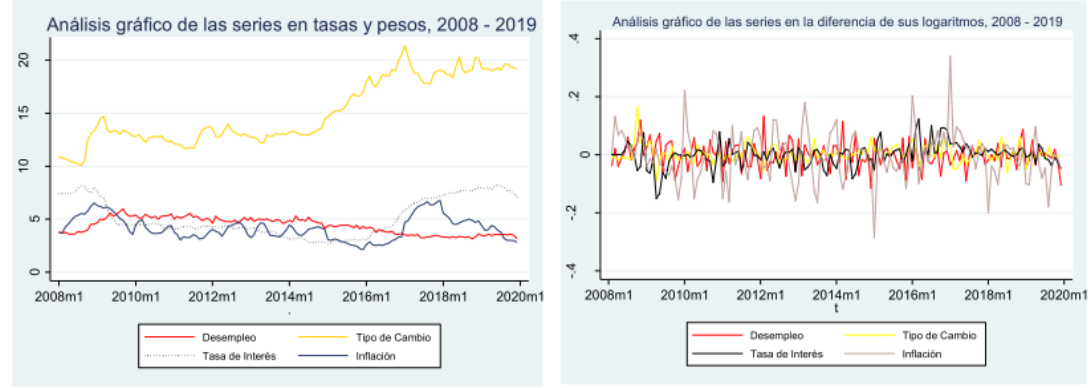

Fuente: Elaboración propia con información del modelo

Las variables empleadas en el modelo se enlistan en tabla 1:

Tabla 1. Características de las variables

\begin{tabular}{|l|l|}
\multicolumn{1}{|c|}{ Variable } & \multicolumn{1}{c|}{ Características } \\
\hline Inflación & $\begin{array}{l}\text { Se empleó la tasa de inflación anual como variable para representar el nivel de } \\
\text { precios prevaleciente en México. }\end{array}$ \\
\hline $\begin{array}{l}\text { Desempleo } \\
\text { Desempleo-ENOE }\end{array}$ & $\begin{array}{l}\text { Representa la tasa de desempleo en México presentada por la OCDE. } \\
\text { Representa la tasa de desempleo en México medida por la ENOE. }\end{array}$ \\
\hline Tipo de cambio & Representa el índice de tipo de cambio promedio de México. \\
\hline Tasas de interés & Es la tasa de interés nominal de los Cetes a 28 días. \\
\hline Postcrisis & $\begin{array}{l}\text { Variable dummy que representa cambios estructurales derivados de la crisis } \\
\text { económica mundial. }\end{array}$ \\
\hline Trump & $\begin{array}{l}\text { Variable dummy que representa los cambios derivados de las elecciones } \\
\text { presidenciales en Estados Unidos. }\end{array}$ \\
\hline Objetivo & $\begin{array}{l}\text { Variable dummy que representa los movimientos en la tasa de interés objetivo } \\
\text { por parte del Banco de México. }\end{array}$ \\
\hline
\end{tabular}

Fuente: Elaboración propia 


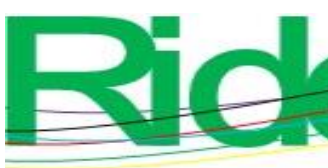

Revista Iberoamericana para la Investigación y el Desarrollo Educativo ISSN 2007 - 7467

\section{Modelo 1. Curva de Phillips para México 1980-2019. Restricciones a largo plazo en un modelo estructural de vectores autorregresivos}

Para el primer modelo se establecen las restricciones a largo plazo para un modelo estructural de vectores autorregresivos para las variables inflación y desempleo, las cuales presentan dos choques (de nuestras variables sobre la otra y sobre sí mismas). Se contempla que la respuesta de las variables a los choques en el largo plazo debe ser cero, ya que las variables son estacionarias. Además, se establece la restricción de que, en el largo plazo, el desempleo no tiene efecto sobre la inflación; por lo tanto, de acuerdo con Schenk (2016), los impulsos respuesta serán cero. De esta manera, el shock "inflshock" se define como aquel que conduce a un cambio en el nivel de la inflación y el shock "desemshock" se define como aquel que no cambia el nivel de inflación en el largo plazo, el cual se presenta de la siguiente forma:

$$
A(L)\left[\begin{array}{c}
\Delta i n f_{t} \\
\operatorname{desem}_{t}
\end{array}\right]=\left[\begin{array}{c}
e_{t^{\text {inflashock }}} \\
e_{t^{\text {desemshock }}}
\end{array}\right]
$$

en donde $\mathrm{L}$ es el operador de retardo y $\mathrm{A}(\mathrm{L})$ es el retardo polinomial. Entonces:

$$
\left[\begin{array}{c}
\Delta \inf _{t} \\
\operatorname{desem}_{t}
\end{array}\right]=C(L)\left[\begin{array}{c}
e_{t^{\text {inflashock }}} \\
e_{t^{\text {desemshock }}}
\end{array}\right]
$$

en donde $\mathrm{C}(\mathrm{L})$ es una matriz de dos por dos que captura las respuestas a largo plazo.

A continuación, se presenta la interpretación de los gráficos que muestran las funciones de impulso y respuesta para las variables de desempleo e inflación para el caso de México, en términos de la tasa de crecimiento de nuestras variables especificadas en el modelo SVAR. En la primera fila se observa que ante un choque del desempleo, la inflación aumenta en los primeros meses, pero baja significativamente durante los siguientes periodos para después retomar su valor estacionario y aumentar un poco. En la fila inferior se observa que ante un choque de la inflación, el desempleo sube inicialmente, vuelve a su valor inicial en el primer periodo, mantiene el mismo comportamiento el segundo periodo y aumenta durante los siguientes tres para mantenerse un poco por arriba de su valor estacionario.

Para el caso de las funciones observadas en sus impulsos acumulados, en la primera parte observamos que la inflación aumenta con el impacto en los primeros periodos y baja para después dirigirse de nuevo a su valor estacionario, comportamiento que se repite en 

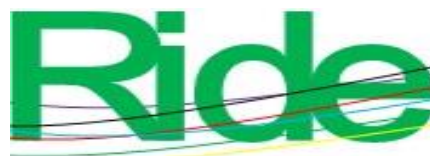

Revista Iberoamericana para la Investigación y el Desarrollo Educativo

ISSN $2007-7467$

segundo caso. Con base en lo anterior, aunque pudiera observarse una reacción momentánea, no se encontraron elementos para asegurar la existencia de la curva de Phillips en el largo plazo para México en el periodo analizado (ver gráficas de funciones de impulso y respuesta, y función de impulso y respuesta acumulada, figura 8).

Figura 8. Funciones de impulso y respuesta y función de impulso y respuesta acumulada para las variables de desempleo e inflación para el caso de México

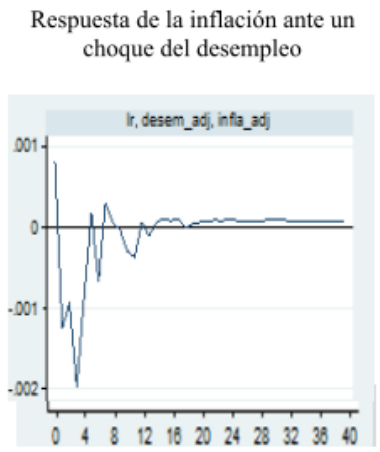

Respuesta de la inflación ante un

Respuesta acumulada de la inflación ante choque del desempleo un choque del desempleo
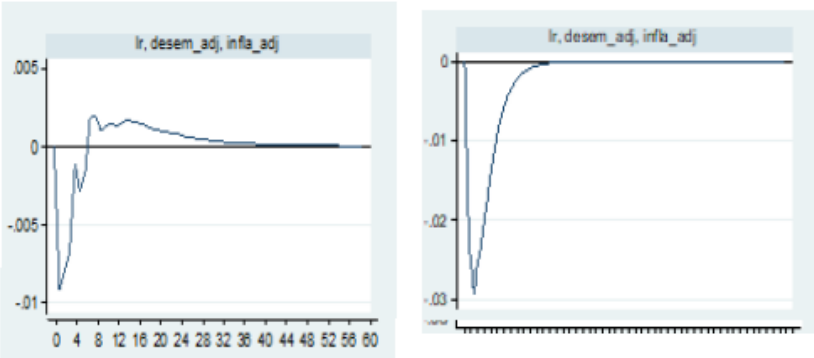

Respuesta del desempleo ante un choque de la inflación

Respuesta del desempleo ante un choque de la inflación

Respuesta acumulada de la inflación ante un choque del desempleo
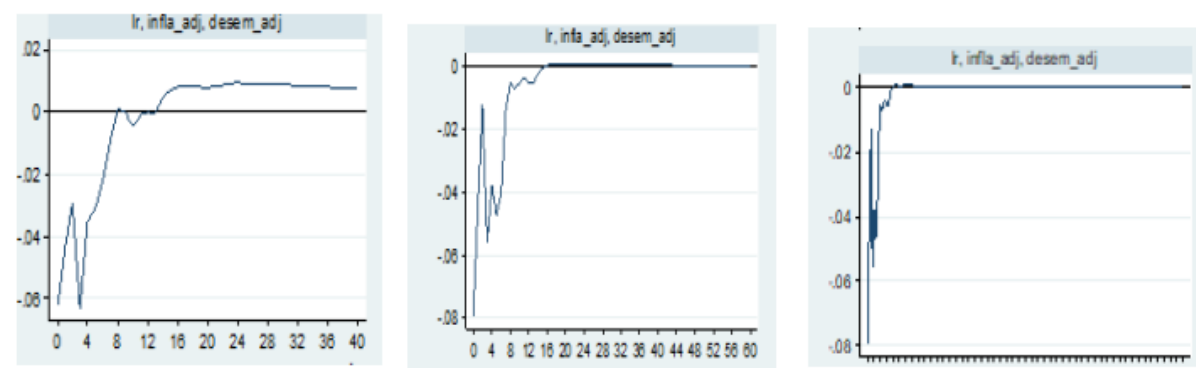

Fuente: Elaboración propia con los datos del modelo planteado 


\section{Modelo 2. Curva de Phillips, tasa de interés y tipo de cambio para México 2008-2019. Modelo estructural de vectores autorregresivos}

En este segundo modelo, se analiza el grado de interdependencia entre la tasa de interés y el tipo de cambio para el caso de México, utilizando el periodo de 2008 a 2019, lapso en el que el Banco de México ha implementado la tasa de interés objetivo como parte de la política monetaria en el país. A continuación, se presentan los resultados de la estimación del modelo SVAR con cuatro ecuaciones y doce rezagos, y se consideran dos cortes estructurales. Uno para el periodo que comprende julio de 2008 a abril de 2009 y otro para el periodo de enero de 2017 a agosto de 2018. Además, el modelo integra una variable dummy para señalar los movimientos en la tasa de interés objetivo; esta variable toma el valor 1 en el caso de los meses en los que esta variable haya sido modificada como parte de la política monetaria del Banco de México.

Para las estimaciones econométricas se empleó información de variables macroeconómicas publicadas por el INEGI y el Banco de México. Las variables fueron obtenidas en tasas y fueron transformadas a logaritmo natural y, en algunos casos, a primeras diferencias. Los datos de las series son mensuales y todas las series cumplen con el principio de estacionariedad y el modelo cumple con las pruebas conjuntas.

El modelo cumple con los supuestos de normalidad, heteroscedasticidad y autocorrelación y es un modelo estable. En cuanto a la normalidad, el modelo no integra la prueba de Jarque-Bera debido a que en la estimación del SVAR se utilizó la herramienta "dfk", la cual es una opción econométrica que ajusta los grados de libertad para muestras pequeñas, dando por supuesto que el principio se cumple. Esta técnica se utiliza en casos como el nuestro en el que se contó con pocos datos para realizar las estimaciones correspondientes (StataCorp, 2013). A través de la prueba de Portmanteau, se logró exponer que las innovaciones contemporáneas al cuadrado se comportan como ruido blanco. Además, de acuerdo con la prueba estadística de multiplicador de Lagrange, no existe autocorrelación entre los residuos del modelo de ecuaciones simultáneas presentado. Finalmente, todos los valores se encontraron dentro del círculo unitario, por lo que el modelo cumple con las condiciones de estabilidad (ver tablas 2, 3 y 4). 


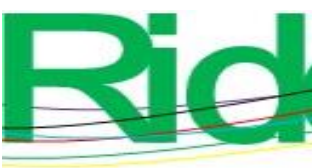

Revista Iberoamericana para la Investigación y el Desarrollo Educativo ISSN 2007 - 7467

Tabla 2. Pruebas Dickey-Fuller-aumentada para variables adicionales

\begin{tabular}{|c|c|c|c|c|c|}
\hline Variable & $\mathrm{Z}(\mathrm{t})$ & $\begin{array}{l}1 \% \text { valor } \\
\text { crítico }\end{array}$ & $\begin{array}{l}5 \% \text { valor } \\
\text { crítico }\end{array}$ & $\begin{array}{l}10 \% \\
\text { valor } \\
\text { crítico }\end{array}$ & $\begin{array}{c}\text { Significanci } \\
\mathrm{a}\end{array}$ \\
\hline Desempleo & 11.104 & 4.026 & 3.445 & 3.145 & $*$ \\
\hline Inflación & 7.729 & 4.026 & 3.445 & 3.145 & $*$ \\
\hline Tipo de cambio & 8.262 & 4.026 & 3.445 & 3.145 & $*$ \\
\hline Tasa de interés & 7.066 & 4.026 & 3.445 & 3.145 & $*$ \\
\hline $\begin{array}{l}* \text { Significativos } \\
\text { y } 10 \%\end{array}$ &, $5 \%$ & & & & \\
\hline
\end{tabular}

Fuente: Estimación elaborada con datos del Inegi y del Banco de México

Tabla 3. Prueba del multiplicador de Lagrange

\begin{tabular}{|c|c|c|}
\hline $\begin{array}{c}\text { Número de } \\
\text { retardo }\end{array}$ & chi $^{2}$ & Prob $>$ chi $^{2}$ \\
\hline 1 & 22.5412 & 0.12656 \\
\hline 2 & 21.5494 & 0.15835 \\
\hline 3 & 15.9867 & 0.45389 \\
\hline 4 & 18.9011 & 0.27383 \\
\hline 5 & 10.9793 & 0.81076 \\
\hline 6 & 17.539 & 0.35157 \\
\hline 7 & 18.8415 & 0.27697 \\
\hline 8 & 13.1109 & 0.66463 \\
\hline 9 & 17.4758 & 0.35547 \\
\hline 10 & 14.0365 & 0.596 \\
\hline 11 & 15.4511 & 0.49188 \\
\hline 12 & 9.3061 & 0.90027 \\
\hline
\end{tabular}

Fuente: Elaboración propia con base en los resultados del modelo

Tabla 4. Prueba estadístico Portmanteau

\begin{tabular}{|l|c|c|}
\hline & Estadístico Portmanteau & $\begin{array}{c}\text { Prob } \\
\text { chi }^{2}\end{array}$ \\
\hline Desempleo & 0.1114 & 0.7385 \\
\hline Inflación & 0.2354 & 0.6275 \\
\hline Tipo de cambio & 0.0117 & 0.9137 \\
\hline Tasa de interés & 0.4791 & 0.4888 \\
\hline
\end{tabular}

Fuente: Elaboración propia con base en el modelo 


\section{Resultados}

A continuación, se presenta la interpretación de los gráficos que muestran las funciones de impulso y respuesta (ver gráficas de impulso-respuesta, figura 9) para las variables desempleo, inflación, tipo de cambio y tasa de interés para el caso de México, esto en términos de la tasa de crecimiento de las variables especificadas en el modelo SVAR. Los valores de los coeficientes representan el cambio porcentual en las variables correspondiente a un impulso.

Figura 9. Funciones impulso respuesta
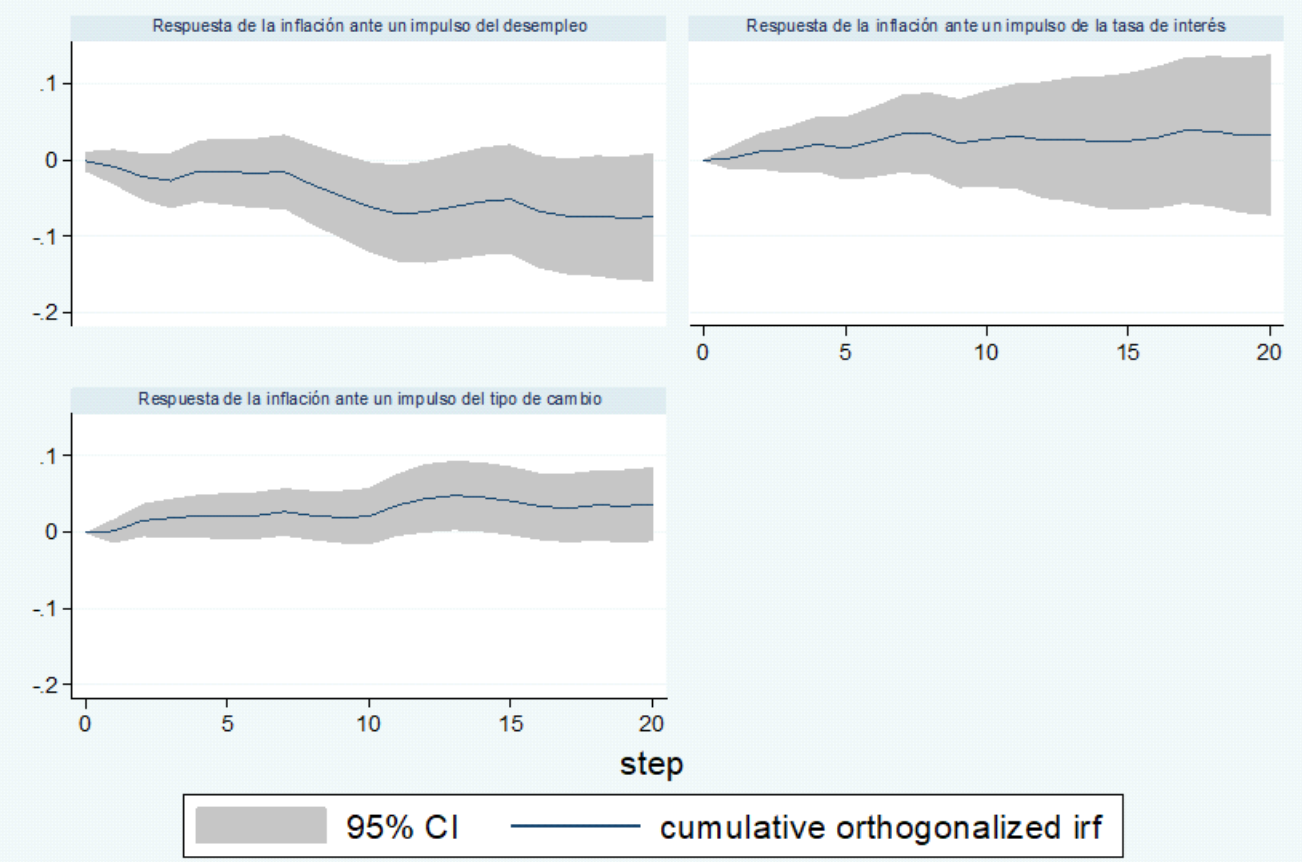

Graphs by irfname, impulse variable, and response variable

Fuente: Elaboración propia con los datos del modelo planteado

De acuerdo con las respuestas observadas, la inflación responde ligeramente ante los choques de las variables analizadas. Ante un impulso del desempleo, la inflación baja marginalmente durante los primeros periodos para después regresar a su media. En cuanto a las respuestas, considerando el impulso de la tasa de interés y de tipo de cambio, se observa que la inflación aumenta y conserva el crecimiento durante varios periodos antes de regresar a su media. Contrario a lo esperado, en el modelo se observa que un choque de la tasa de interés aumenta ligeramente la inflación, aunque en el largo plazo se acerque nuevamente a su media. 


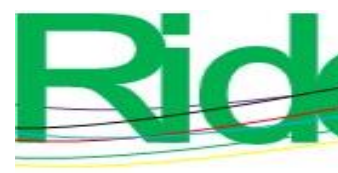

Revista Iberoamericana para la Investigación y el Desarrollo Educativo ISSN 2007 - 7467

En este documento se presentaron dos modelos SVAR para analizar la curva de Phillips en México. En el primero, se analizó un modelo de dos ecuaciones, enfocado en analizar las restricciones a largo plazo en un modelo estructural de vectores autorregresivos en el que se observa que la curva no se presenta en el largo plazo. En el segundo, se hizo un modelo SVAR de cuatro ecuaciones en el que además de las variables de desempleo en inflación, se utilizaron las variables tasa de interés y tipo de cambio. Los resultados del segundo modelo mostraron una curva de Phillips débil y en la que se observa una mayor respuesta de la inflación ante choques dados por el tipo de cambio y la tasa de interés que del desempleo.

\section{Conclusiones}

La curva de Phillips mide la relación de variables fundamentales de una economía. Por esta razón, el debate respecto a esta ha marcado una polémica desde sus orígenes. La propuesta original de dicha curva y su evolución teórica ha desarrollado el análisis de la inflación y el desempleo como variables determinantes para la toma de decisiones de política económica.

Con la estimación del modelo SVAR con las variables inflación y desempleo, se mostró que la curva de Phillips para México se presenta solo en el corto plazo. Cuando se estimó el modelo, incluyendo las variables tasa de interés y tipo de cambio, se comprobó que estas son las que explican los movimientos en la inflación.

En México, el banco central ha aplicado una política monetaria restrictiva con el objetivo de mantener estable el nivel de precios, particularmente, la tasa de interés y el tipo de cambio.

La estrecha relación que existe entre la sobrevaluación del tipo de cambio y el bajo nivel inflacionario muestran que esta estrategia es la que se ha convertido en el principal mecanismo de control de precios, particularmente de la inflación.

Por todo esto, y a diferencia de algunos estudios, se demostró que la curva de Phillips no se cumple para el caso de México en el largo plazo, en el periodo de estudio 1980-2019. 


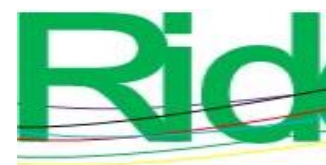

Revista Iberoamericana para la
Investigación y el Desarrollo Educativo
ISSN $2007-7467$

Futuras líneas de investigación

A pesar de que no hay modelos que comprueben la existencia de la curva de Phillips, el banco central mexicano sigue tomando en cuenta esta herramienta en su manejo de política monetaria, en el que su principal objetivo es el control inflacionario. Sin embargo, en el último año, debido a la crisis económica generada por la covid-19, los bancos centrales han adoptado políticas expansivas, con incrementos en estímulos fiscales, tasas de interés relajadas y permitiendo una tasa inflacionaria más alta.

Esto nos lleva a considerar que el Banco de México debe dejar de lado que una mayor tasa de empleo va a aumentar los niveles de precios, lo que daría pie a que el banco central tuviera un doble mandato no solo con especial atención en la inflación, sino también en la búsqueda de un pleno empleo. Lo anterior da pie a las siguientes líneas de investigación:

- Determinantes de la inflación en México en un contexto de control inflacionario, 1994-2021.

- Keynesianos, postkeynesianos y neokeynesianos en México, un análisis de las metodologías y resultados, 2000-2020.

- Expectativas de inflación en México: análisis de las palabras en los anuncias y minutas de política monetaria, 1994-2021.

- Adaptación de las políticas monetarias durante la crisis de la covid-19, experiencias internacionales de México, Estados Unidos y Canadá, 2019-2021. 


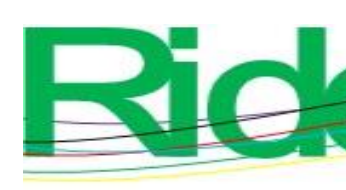

Revista Iberoamericana para la Investigación y el Desarrollo Educativo ISSN $2007-7467$

\section{Referencias}

Amisano, G. and Giannini, C. (1997). Topics in Structural VAR Econometrics ( $2^{\text {th }}$ ed.). Heidelberg: Springer.

BALL, L. and MANKIW, G. M. (2002). The NAIRU in theory and practice. The Journal of Economic Perspectives, 16(4), Autumn, 115-136.

Calvo, G. (1983). Staggered prices in a utility-maximizing framework. Journal of Monetary Economics, 12(3), 983-998.

Colombo, L. and Weinrich, G. (2003). The Phillips Curve as a long run phenomenon in a macroeconomic modelo with complex dynamics. Journal of Economic Dynamics \& Control, 28(1), 1-26.

Cruz, F. (2006). La política monetaria y el tipo de cambio: el mecanismo de estabilidad monetaria en México (1988-2005) (tesis de maestría). México: FES-Acatlán.

Dickey, D. A. and Fuller, W. A. (1981). Likelihood Ratio Statistics for Autoregressive Time Series with a Unit Root. Econometrica, (49).

Dorta, M., Álvarez, F. y Zavarce, H. (2001). Curva de Phillips de mediano plazo. Venezuela: Banco Central de Venezuela. Oficina de Consultoría Económica.

Engle, R. and Granger, W. (1987) Cointegration and Error Correction: Representation, Estimation and Testing. Econometrica, 55:251-276.

Forni, M. and Gambetti, L. (2014). Sufficient information in structural VARs. Journal of Monetary Economics, 66(C), 124-136.

Friedman, M. (1967); "The Role of Monetary Policy"; American Economic Review, marzo, pp. 1- 17.

Laguna, Ch. (2007). Dinámica inflacionaria y brecha en la producción. La curva de Phillips en México. Análisis Económico, 22(50), 121-147.

Levy, N. (2001). Cambios institucionales del sector financiero y su efecto sobre el fondeo de la inversión en México, 1960-1994. México: Facultad de Economía UNAM.

Lipsey, R. G. (1960). The relation between unemployment and the rate of change of Money wage rates in the United Kingdom, 1862-1957: A further analysis. Economica, 27, 283-299.

López, E. y Misas, M. (1999). Un examen empírico de la curva de Phillips en Colombia. Colombia: Banco de la República de Colombia.

Loria, E., Valdez, J. y Tirado, R. (2019). Estimación de la NAIRU para México, 2002Q12018Q2. Investigación Económica, 78(308). 

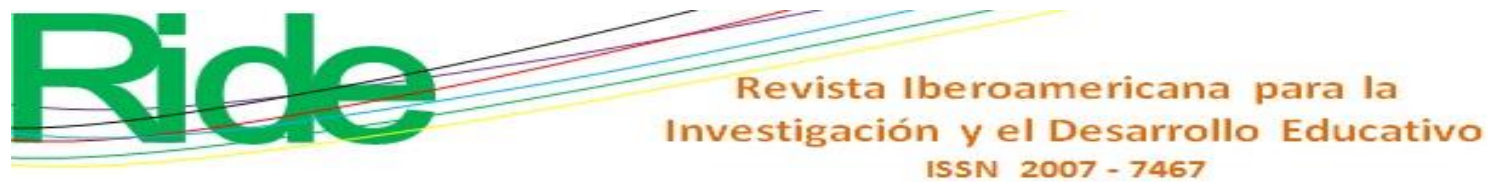

Lucas, R. (1973). Econometric testing of the natural rate hypothesis. In Eckstein, O. (ed.), The econometrics of price determination conference. Board of Governors of the Federal Reserve System y Social Research Council, Washington D.C.

Lucas, R. (1973). Some international evidence on output-inflation trades off. American Economic Review, (63), 326-334.

Mankiw, N. (2001). The inexorable and mysterious trade-off between inflation and unempleoyment. Economic Journal (111), 45-61.

Mántey, G. (1997). Lecciones de economía monetaria. México: Ed. UACP y P-UNAMAcatlán.

Mántey, G. (2005). La política monetaria en México y la tasa de interés real. Investigación Económica, (211).

Morales, A. (2004). La inflación en la zona del euro: un análisis desde el lado de la oferta. España: Sector Exterior Español.

Muñoz, E., Rojas, Sáenz y Tenorio, E. (2003). La curva de Phillips en Costa Rica. Banco Central de Costa Rica. Departamento de Investigaciones Económicas. Documento de Investigación.

Muth, J. F. (1961). Rational Expectations and the Theory of Price Movements. Econometrica, 29(3), 315-335.

Okun, A. (1962). Potential GNP \& Its Measurement and Significance. American Statistical Association, Proceedings of the Business and Economics Statistics Section, 98-104.

Phelps, E. (1968). Money-Wage dynamic and labor-market equilibrium. Journal of Political Economy, 76(S4).

Phillips, A. W. (1958). The relation between unemployment and the rate of change of money wages in the United Kingdom, 1861-1957, Economica, 25(100), 283-299.

Ramos, R. (2000). Objetivos e instrumentos de política económica: una aplicación a México. México: Fondo de Cultura Económica.

Rico, P. (1999). El efecto Fisher y la paridad de interés real. Evidencia para la economía española. España: Instituto Valenciano de Investigaciones Económicas A.C. Universidad de Valencia.

Rodríguez, P., Ludlow, J. y Peredo. F. (2004). La curva de Phillips y la NAIRU en México. Economía, Teoría y Práctica, (20), pp. 83-102.

Ros, J (1979). Inflación: la experiencia de la década de los setenta. Economía Mexicana, (1). 


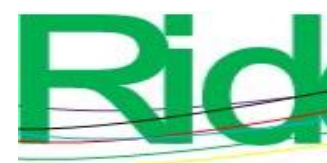

Revista Iberoamericana para la
nvestigación y el Desarrollo Educativo
ISSN $2007-7467$

Samuelson, P. and Solow, R. (1960). Analytical aspects of Anti-inflation Policy. American Review.

Sargent, T. J. (1973). Rational expectations, the real rule of interest, and the "natural" rate of unemployment. Brookings papers on Economic Activity, 2, 429-472.

Sargent, T. J. and Wallace, N. (1975). Rational expectations the optimal money instrument and the optimal money supply rule. Journal of Political Economy, 83(2), 241-254

Schaling, E. (1999). The nonlinear Phillips Curve and inflation forecast targeting. Journal of Money, Credit and Banking, 36(3), 361-386.

Schenck, D. (2016). Structural vector autoregression models in Stata. Retrieved from https://blog.stata.com/2016/09/20/structural-vector-autoregression-models/

StataCorp. 2013. Stata 13 Base Reference Manual. College Station, TX: Stata Press. Retrieved from https://www.stata.com/manuals/tsvar.pdf

Tobin, J. (1972). The Wage-Price Mechanism Overview of the Conference. In Eckstein, O. (ed.), The econometrics of price determination conference. Washington D. C.: Federal Reserve System. 


\section{Revista Iberoamericana para la Investigación y el Desarrollo Educativo ISSN 2007 - 7467}

\begin{tabular}{|l|l|}
\hline Rol de contribución & Autor(es) \\
\hline Conceptualización & Ericka J. Arias Guzmán \\
\hline Metodología & $\begin{array}{l}\text { Ericka J. Arias Guzmán, Felipe Cruz Díaz } \\
\text { y Karla Barcellos (igual) }\end{array}$ \\
\hline Software & No aplica \\
\hline Validación & No aplica \\
\hline Análisis formal & Karla Barcellos \\
\hline Investigación & $\begin{array}{l}\text { Ericka J. Arias Guzmán, Felipe Cruz Díaz } \\
\text { y Barcellos (igual) }\end{array}$ \\
\hline Recursos & No aplica \\
\hline Curación de datos & Karla Barcellos \\
\hline $\begin{array}{l}\text { Escritura- preparación del } \\
\text { borrador original }\end{array}$ & Ericka J. Arias Guzmán \\
\hline $\begin{array}{l}\text { Visualización } \\
\text { Supervisión }\end{array}$ & $\begin{array}{l}\text { Ericka J. Arias Guzmán, Felipe Cruz Díaz } \\
\text { y Barcellos (igual) }\end{array}$ \\
\hline $\begin{array}{l}\text { Administración de } \\
\text { proyectos }\end{array}$ & Ericka J. Arias Guzmán \\
\hline Adquisición de fondos & Ericka J. Arias Guzmán \\
\hline
\end{tabular}

